\title{
Боковой перенос энергии при возбуждении плазмонов терагерцовой волной в периодической пространственно несимметричной графеновой структуре
}

\author{
(С) Д.В. Фатеев ${ }^{1,2}$, К.В. Машинский ${ }^{1}$, И.М. Моисеенко ${ }^{1}$, В.В. Попов ${ }^{1}$ \\ ${ }^{1}$ Саратовский филлиал Института радиотехники и электроники им. В.А. Котельникова Российской академии наук, \\ 410019 Саратов, Россия \\ ${ }^{2}$ Саратовский государственный университет им. Н.Г. Чернышевского, \\ 410012 Саратов, Россия \\ E-mail: FateevDV@yandex.ru
}

Поступила в Редакцию 24 апреля 2019 г.

В окончательной редакции 29 апреля 2019 г.

Принята к публикации 29 апреля 2019 г.

\begin{abstract}
Теоретически исследовано преобразование мощности терагерцовой волны, нормально падающей на периодическую графеновую структуру, в мощность бегущей плазменной волны. Найдены режимы максимального преобразования мощности падающего излучения в мощность бегущего плазмона и режим возбуждения однонаправленно бегущего плазмона. Выяснено, что до 15\% мощности падающей волны может преобразоваться в мощность бегущего плазмона.
\end{abstract}

Ключевые слова: плазмон, терагерцовое излучение, графен, бегущая волна.

DOI: 10.21883/FTP.2019.09.48122.05

\section{1. Введение}

В последнее время интенсивно исследуются плазмонные свойства графена в терагерцовом (ТГц) частотном диапазоне [1-3]. В настоящее время современные технологии позволяют создавать графеновые структуры с подвижностью носителей заряда $\sim 100000 \mathrm{~cm}^{2} /(\mathrm{B} \cdot \mathrm{c})$ и временем релаксации импульса носителей $\sim \tau=1$ пс при комнатных температурах [4], что соответствует теоретическим предсказаниям $[5,6]$. Указанные значения времени релаксации импульса позволяют возбуждать плазмонные резонансы на ТГц частотах.

Обычно для возбуждения плазмонных резонансов в графене используются короткопериодические решетки, позволяющие связать медленные дифракционные гармоники электрического поля [7] с медленными плазмонными модами в графене [8]. При таком способе возбуждения, как правило, возбуждаются стоячие плазменные волны. Для возбуждения бегущих плазменных волн в графене используются дифракция электромагнитной волны на одиночных объектах, таких как: 1) острие сканирующего ближнеполевого микроскопа [9], одиночный металлический затвор, расположенный над графеном [10], щель в металлическом экране, расположенном над графеном [11]. Еще одним способом возбуждения бегущего плазмона является использование постоянного дрейфа в графене в периодических структурах [12]. Наконец следует упомянуть способы возбуждения бегущего плазмона в графене с помощью эффекта нарушенного полного внутреннего отражения с использование призм [13,14].

В данной работе исследуется преобразование ТГц волны, нормально падающей на периодическую графеновую структуру с асимметричной элементарной ячейкой, в бегущую плазменную волну в графене.

\section{2. Постановка задачи и метод решения}

Рассматриваемая структура (рис. 1) состоит из графена, над которым расположен двойной решеточный металлический затвор с асимметричной элементарной ячейкой. Структура расположена на полубесконечной диэлектрической подложке. Терагерцовая волна, с поляризацией электрического поля поперек полосок решетки, падает на решеточный затвор и возбуждает плазменные колебания в графене. За счет создания геометрической асимметрии элементарной ячейки графеновой структуры возможно возбуждение плазменной волны с неравными встречными пространственными фурье-гармониками порядков $+p$ и $-p$ [15], что может приводить к возбуждению бегущей плазменной волны и к возникновению эффектов увлечения носителей заряда

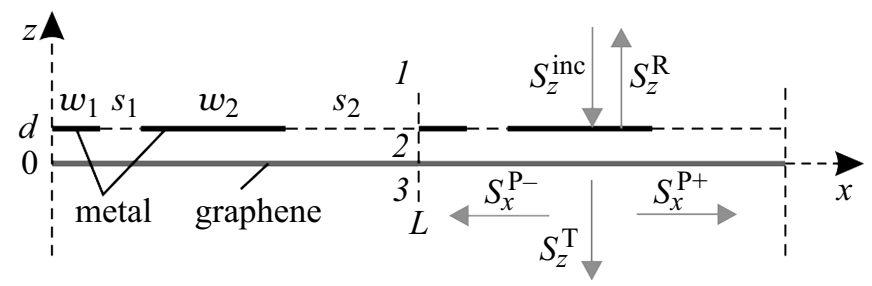

Рис. 1. Схематическое изображение периодической графеновой структуры с двойным решеточным затвором (показаны две элементарные ячейки). На рисунке справа показаны потоки мощности в структуре. 
в графене бегущей плазменной волной $[15,16]$. В расчетах использовалось значение энергии Ферми носителей заряда в графене 150 мэВ.

Для моделирования возбуждения бегущей плазменной волны в периодической структуре была решена электродинамическая задача о нормальном падении однородной ТГц электромагнитной волны на рассматриваемую структуру. На первом этапе электродинамического подхода уравнения Максвелла в трех диэлектрических средах записываются в фурье-представлении, с разложением всех компонент индуцированных электрического и магнитного полей в ряды Фурье как

$$
E_{x}(x, z, t)=\exp (-i \omega t) \sum_{p=-\infty}^{\infty} e_{x, p}(z) \exp \left(i q_{p} x\right),
$$

где $E_{x}(x, z, t)-x$-компонета электрического поля, $e_{x, p}(z)$ - фурье-амплитуды и $q_{p}=2 \pi p / L-$ векторы обратной решетки, $p$ - целое число и $L-$ пространственный период графеновой структуры. Зависимость индуцированных электрических полей от координаты $\mathrm{z}$ в диэлектрических средах записывалась в виде

$$
\begin{aligned}
& e_{x, p}^{(1)}(z)=e_{x, p}^{(1)} \exp \left(i q_{z, p}^{(1)} z\right), \\
& e_{x, p}^{(2)}(z)=e_{x, p}^{(2,1)} \exp \left(i q_{z, p}^{(2)} z\right)+e_{x, p}^{(2,2)} \exp \left(-i q_{z, p}^{(2)} z\right), \\
& e_{x, p}^{(3)}(z)=e_{x, p}^{(3)} \exp \left(i q_{z, p}^{(3)} z\right),
\end{aligned}
$$

где $e_{x, p}^{(\alpha)}(z) \quad$ - фурье-амплитуды электрического поля в различных диэлектрических средах, $q_{z, p}^{(\alpha)}= \pm \sqrt{\omega^{2} \varepsilon_{\alpha} \varepsilon_{0} \mu_{0}-q_{P}^{2}}-$ поперечные волновые числа пространственных фурье-гармоник и $\alpha=1,2,3-$ номер среды. Связь электрических и магнитных полей в различных диэлектрических средах описывается граничными условиями в плоскостях $z=d$ и $z=0$, в виде

$$
\begin{aligned}
& \left\{\begin{array}{l}
e_{x, p}^{(1)}(d)+\delta_{p, 0} E_{x}^{\mathrm{inc}}(d)=e_{x, p}^{(2)}(d), \\
h_{y, p}^{(1)}(d)+\delta_{p, 0} H_{y}^{\mathrm{inc}}(d)-h_{y, p}^{(2)}(d)=-j_{x, p}(d),
\end{array}\right. \\
& \left\{\begin{array}{l}
e_{x, p}^{(2)}(0)=e_{x, p}^{(3)}(0), \\
h_{y, p}^{(2)}(0)-h_{y, p}^{(3)}(0)=-\sigma_{\mathrm{gr}}(\omega) e_{x, p}^{(2)}(0),
\end{array}\right.
\end{aligned}
$$

где $j_{x, p}(d)$ - фурье-амплитуды электрического тока в плоскости металлического затвора $z=d, \sigma_{\mathrm{gr}}(\omega)-$ проводимость графена, описываемая формулой $[17,18]$

$$
\begin{gathered}
\sigma_{\mathrm{gr}}(\omega)=\frac{e^{2} 2 \tau k_{\mathrm{B}} T}{\pi \hbar^{2}(1-i \omega \tau)} \ln \left(2 \operatorname{ch}\left(\frac{\varepsilon_{\mathrm{F}}}{2 k_{\mathrm{B}} T}\right)\right) \\
+\frac{e^{2}}{4 \hbar^{2}} Q\left(\frac{\hbar \omega}{2}\right)-\frac{e^{2} \omega}{i \pi} \int_{0}^{\infty} \frac{Q(\varepsilon)-Q\left(\frac{\hbar \omega}{2}\right)}{\hbar^{2} \omega^{2}-4 \varepsilon^{2}} d \varepsilon, \\
Q(\vartheta)=\frac{\operatorname{sh}\left(\frac{\vartheta}{k_{\mathrm{B}} T}\right)}{\operatorname{ch}\left(\frac{\vartheta}{k_{\mathrm{B}} T}\right)+\operatorname{ch}\left(\frac{\varepsilon_{\mathrm{F}}}{k_{\mathrm{B}} T}\right)} .
\end{gathered}
$$

Здесь $\varepsilon_{\mathrm{F}}$ - энергия Ферми в графене, $e-$ заряд электрона, $k_{\mathrm{B}}$ - постоянная Больцмана и $T-$ температура.
С использованием уравнений Максвелла и граничных условий в фурье-представлении можно записать соотношение между фурье-амплитудами электрического поля и плотности тока в плоскости $z=d$

$$
\begin{aligned}
e_{x, p}^{(1)}(d) & =Z_{P}\left(\sigma_{\mathrm{gr}}\right) j_{d} \\
- & \left(2 \sqrt{\frac{\varepsilon_{0} \varepsilon_{1}}{\mu_{0}}} Z_{0}\left(\sigma_{\mathrm{gr}}\right) e^{-i d \omega \sqrt{\varepsilon_{0} \mu_{0} \varepsilon_{1}}}+1\right) \delta_{p, 0} E_{x}^{\text {inc }},
\end{aligned}
$$

где

$$
Z_{p}\left(\sigma_{\mathrm{gr}}\right)=-\frac{q_{z, 0}^{(1)} q_{z, 0}^{(2)}\left(t_{2} q_{z, 0}^{(3)} \varepsilon_{0} \varepsilon_{2} \omega-t_{1} q_{z, 0}^{(2)} t_{3}\right)}{\varepsilon_{0} \chi \omega}
$$

- фурье-импедансы графеновой структуры в плоскости решеточного затвора, при получении которых использовались следующие величины:

$$
\begin{gathered}
\chi=\left(\xi_{2} q_{z, 0}^{(1)} \varepsilon_{2}-\xi_{1} q_{z, 0}^{(2)} \varepsilon_{1}\right) q_{z, 0}^{(2)} \xi_{3}+\left(\xi_{2} q_{z, 0}^{(2)} \varepsilon_{1}\right. \\
\left.-\xi_{1} q_{z, 0}^{(1)} \varepsilon_{2}\right) \varepsilon_{0} \omega q_{z, 0}^{(3)} \varepsilon_{2}, \\
\xi_{1}=-1+e_{z, 0}^{2 i d q^{(2)}}, \quad \xi_{2}=1+e_{z, 0}^{2 i d q^{(2)}}, \quad \xi_{3}=q_{z, 0}^{(3)} \sigma_{\mathrm{gr}}-\varepsilon_{0} \varepsilon_{3} \omega .
\end{gathered}
$$

На втором этапе электродинамического подхода формируются интегральные уравнения относительно осциллирующих токов в плоскости $z=d$. Для этого используется закон Ома в виде

$$
I_{x}(x, d)=\sigma(x, d) E_{x}(x, d),
$$

где $I_{x}(x, d)=\sigma(x, d) E_{x}(x, d)$ - плотность электрического тока в плоскости решеточного затвора, и

$$
\sigma(x, d)= \begin{cases}\sigma_{\mathrm{M}} & \text { при } 0<x<w_{1}, \\ 0 & \text { при } w_{1}<x<w_{1}+w_{2}, \\ \sigma_{\mathrm{M}} & \text { при } w_{1}+w_{2}<x<w_{1}+w_{2}+w_{3}, \\ 0 & \text { при } w_{1}+w_{2}+w_{3}<x<L,\end{cases}
$$

где $\sigma_{\mathrm{M}}-$ поверхностная проводимость металла. Используя выражения (4)-(6) и обратное преобразование Фурье для плотности электрического тока, можно записать два связанных интегральных уравнения относительно плотностей электрических токов на затворных электродах элементарной ячейки решеточного затвора:

$$
\begin{aligned}
& I_{x}^{\left(w_{1}\right)}(x, d)-\sigma_{\mathrm{M}} 2 \sqrt{\frac{\varepsilon_{0} \varepsilon_{1}}{\mu_{0}}} \exp \left(-i \omega d \sqrt{\varepsilon_{0} \mu_{0} \varepsilon_{1}}\right) Z_{0} E_{x}^{\mathrm{inc}} \\
& =\int_{0}^{w_{1}} I_{x}^{\left(w_{1}\right)}\left(x^{\prime}, d\right) G\left(x, x^{\prime}\right) d x^{\prime}+\int_{w_{1}+s_{1}}^{w_{1}+s_{1}+w_{2}} I_{x}^{\left(w_{2}\right)}\left(x^{\prime}, d\right) G\left(x, x^{\prime}\right) d x^{\prime}, \\
& I_{x}^{\left(w_{2}\right)}(x, d)-\sigma_{\mathrm{M}} 2 \sqrt{\frac{\varepsilon_{0} \varepsilon_{1}}{\mu_{0}}} \exp \left(-i \omega d \sqrt{\varepsilon_{0} \mu_{0} \varepsilon_{1}}\right) Z_{0} E_{x}^{\mathrm{inc}} \\
& =\int_{0}^{w_{1}} I_{x}^{\left(w_{1}\right)}\left(x^{\prime}, d\right) G\left(x, x^{\prime}\right) d x^{\prime}+\int_{w_{1}+s_{1}}^{w_{1}+s_{1}+w_{2}} I_{x}^{\left(w_{2}\right)}\left(x^{\prime}, d\right) G\left(x, x^{\prime}\right) d x^{\prime},
\end{aligned}
$$


где $I_{x}^{\left(w_{1}\right)}(x, d)$ - плотность тока на затворном электроде с шириной $w_{1}, I_{x}^{\left(w_{2}\right)}(x, d)$ - плотность тока на затворном электроде с шириной $w_{2}$ и

$$
G\left(x, x^{\prime}\right)=\frac{1}{L} \sigma_{\mathrm{M}} \sum_{p=-\infty}^{\infty} Z_{P}\left(\sigma_{\mathrm{gr}}\right) \exp \left(i q_{P}\left(x-x^{\prime}\right)\right)
$$

- ядра интегралов.

На следующем этапе электродинамического подхода система интегральных уравнений (7) решается методом Галеркина с помощью разложения плотностей электричесекого тока в ряды по ортогональным полиномам Лежандра:

$$
\begin{aligned}
& I_{x}^{\left(w_{1}\right)}(x, d)=\sum_{\beta=0}^{\infty} C_{\beta}^{(1)} P_{\beta}\left(\frac{2 x}{w_{1}}-1\right), \\
& I_{x}^{\left(w_{2}\right)}(x, d)=\sum_{\beta=0}^{\infty} C_{\beta}^{(2)} P_{\beta}\left(2 \frac{x-w_{1}-s_{1}}{w_{2}}-1\right),
\end{aligned}
$$

где $C_{\beta}^{(n)}-$ коэффициенты разложения и $P_{\beta}-$ полиномы Лежандра.

Подстановка выражений (8) в (7) преобразует систему интегральных уравнений в систему алгебраических уравнений относительно неизвестных коэффициентов $C_{\beta}^{(n)}$, которая решается численно. Решение алгебраической системы позволяет вычислить плотности токов на решеточном затворе, используя выражения (8), и электрические поля и токи во всех точках элементарной ячейки графеновой структуры с помощью выражений (4), граничных условий (2) и уравнений Максвелла.

Для вычисления потоков энергии в графеновой структуре использован усредненный по времени вектор Умова-Пойнтинга $\mathbf{S}=0.5 \operatorname{Re}\left[\mathbf{E H}^{*}\right]$, где $\mathbf{E}-$ вектор электрического поля и $\mathbf{H ~ - ~ в е к т о р ~ м а г н и т н о г о ~ п о - ~}$ ля. Поскольку при выбранной поляризации падающей волны будут возбуждаться только ТМ дифракционные поля с компонентами $\left(E_{x}, E_{z}, H_{y}\right)$, то вектор потока Умова-Пойнтинга будет иметь только две компоненты

$$
\begin{aligned}
& S_{x}=-\frac{1}{2} \operatorname{Re}\left(E_{z} H_{y}^{*}\right), \\
& S_{z}=\frac{1}{2} \operatorname{Re}\left(E_{x} H_{y}^{*}\right) .
\end{aligned}
$$

Вычислим поток мощности падающей волны как

$$
S_{z}^{\text {inc }}=-\frac{1}{2} \sqrt{\frac{\varepsilon_{1} \varepsilon_{0}}{\mu_{0}}}\left|E_{x}^{\text {inc }}\right|^{2},
$$

поток мощности отраженной от графеновой структуры волны как

$$
S_{z}^{\mathrm{R}}=\frac{1}{2} \sqrt{\frac{\varepsilon_{1} \varepsilon_{0}}{\mu_{0}}}\left|e_{x, 0}^{(1)}\right|^{2}
$$

и поток мощности прошедшей через графеновую структуру волны как

$$
S_{z}^{\mathrm{T}}=-\frac{1}{2} \sqrt{\frac{\varepsilon_{3} \varepsilon_{0}}{\mu_{0}}}\left|e_{x, 0}^{(3)}\right|^{2} .
$$

Тогда коэффициент поглощения ТГц волны в графеновой структуре может быть записан как

$$
A=1-\frac{S_{z}^{T}+S_{z}^{R}}{S_{z}^{\text {inc }}} .
$$

Усредненный по пространственному периоду структуры поток электромагнитной мощности вдоль графена (в $x$-направлении), возникающий в результате возбуждения бегущей плазменной волны, может выть вычислен как

$$
S_{x}^{P}=\frac{1}{L} \int_{-\infty}^{+\infty} \int_{0}^{L} S_{x} d x d z
$$

Интегрирование по координате $z$ учитывает вклад всех высших эванесцентных фурье-гармоник электромагнитного поля плазмона. Поток мощности плазмона (9) может быть записан как сумма двух встречных потоков $S_{x}^{P}=S_{x}^{P+}+S_{x}^{P-}$, один из которых $\left(S_{x}^{P+}\right)$ направлен в положительном направлении оси $x$, а другой в отрицательном $\left(S_{x}^{P-}\right)$, при этом каждый из них должен суммировать потоки во всех трех средах

$$
\begin{aligned}
& S_{x}^{P+}=S_{x}^{P 1+}+S_{x}^{P 2+}+S_{x}^{P 3+}, \\
& S_{x}^{P-}=S_{x}^{P 1-}+S_{x}^{P 2-}+S_{x}^{P 3-} .
\end{aligned}
$$

Вклады потоков Умова-Пойнтинга различных направлений в различных средах вычисляются с помощью следующих выражений:

$$
\begin{gathered}
S_{x}^{P 1+}=\frac{\omega \varepsilon_{1} \varepsilon_{0}}{4} \sum_{p>0} \frac{q_{p}}{\left|q_{z, p}^{(1)}\right|^{2}} \frac{\beta_{p}^{(1)}\left|e_{x, p}^{(1)}\right|^{2}}{\operatorname{Im}\left(q_{z, p}^{(1)}\right)} \\
S_{x}^{P 2+}=\frac{\omega \varepsilon_{2} \varepsilon_{0}}{4} \sum_{p>0} \frac{q_{p}}{\left|q_{z, p}^{(2)}\right|^{2}} \frac{\beta_{p}^{(2,2)}\left|e_{x, p}^{(2,2)}\right|^{2}-\beta_{p}^{(2,1)}\left|e_{x, p}^{(2,1)}\right|^{2}}{\operatorname{Im}\left(q_{z, p}^{(2)}\right)} \\
-\omega \varepsilon_{2} \varepsilon_{0} d \sum_{p>0} \frac{q_{p}}{\left|q_{z, p}^{(2)}\right|^{2}} \operatorname{Re}\left(e_{x, p}^{(2,1)}\left(e_{x, p}^{(2,2)}\right)^{*}\right), \\
S_{x}^{P 3}=\frac{\omega \varepsilon_{3} \varepsilon_{0}}{4} \sum_{p>0} \frac{q_{p}}{\left|q_{z, p}^{(3)}\right|^{2}} \frac{\left|e_{x, p}^{(3)}\right|^{2}}{-\operatorname{Im}\left(q_{z, p}^{(3)}\right)}
\end{gathered}
$$

и

$$
\begin{gathered}
S_{x}^{P 1-}=\frac{\omega \varepsilon_{1} \varepsilon_{0}}{4} \sum_{p<0} \frac{q_{p}}{\left|q_{z, p}^{(1)}\right|^{2}} \frac{\beta_{p}^{(1)}\left|e_{x, p}^{(1)}\right|^{2}}{\operatorname{Im}\left(q_{z, p}^{(1)}\right)} \\
S_{x}^{P 2-}=\frac{\omega \varepsilon_{2} \varepsilon_{0}}{4} \sum_{p<0} \frac{q_{p}}{\left|q_{z, p}^{(2)}\right|^{2}} \frac{\beta_{p}^{(2,2)}\left|e_{x, p}^{(2,2)}\right|^{2}-\beta_{p}^{(2,1)}\left|e_{x, p}^{(2,1)}\right|^{2}}{\operatorname{Im}\left(q_{z, p}^{(2)}\right)} \\
-\omega \varepsilon_{2} \varepsilon_{0} d \sum_{p<0} \frac{q_{p}}{\left|q_{z, p}^{(2)}\right|^{2}} \operatorname{Re}\left(e_{x, p}^{(2,1)}\left(e_{x, p}^{(2,2)}\right)^{*}\right), \\
S_{x}^{P 3-}=\frac{\omega \varepsilon_{3} \varepsilon_{0}}{4} \sum_{p<0} \frac{q_{p}}{\left|q_{z, p}^{(3)}\right|^{2}} \frac{\left|e_{x, p}^{(3)}\right|^{2}}{-\operatorname{Im}\left(q_{z, p}^{(3)}\right)}
\end{gathered}
$$


где

$$
\begin{aligned}
& \beta_{p}^{(1)}=\exp \left(-2 \operatorname{Im}\left(q_{z, p}^{(1)}\right) d\right) \\
& \beta_{p}^{(2, n)}=\exp \left((-1)^{n} 2 \operatorname{Im}\left(q_{z, p}^{(2)}\right) d\right)-1 .
\end{aligned}
$$

Для описания эффективности преобразования мощности падающей ТГц волны в бегущую плазменную волну вводится коэффициент $T_{P}=S_{x}^{P} /\left|S_{L, x}^{\text {inc }}\right|$, где $S_{L, x}^{\text {inc }}=L S_{z}^{\text {inc }}-$ плотность потока падающей волны на элементарную ячейку периодической структуры. Тогда коэффициент преобразования $T_{P}^{+}=S_{x}^{P+} /\left|S_{L, z}^{\text {inc }}\right|$ будет описывать преобразование падающей электромагнитной волны в плазмон, переносящий энергию в положительном направлении оси $x$, а коэффициент преобразования $T_{P}^{-}=S_{x}^{P-} /\left|S_{L, z}^{\text {inc }}\right|$ будет соответствовать отрицательному направлению оси $x$.

\section{3. Результаты расчетов и их обсуждение}

При расчетах использовались следующие параметры графеновой структуры: $w_{2}=0.5$ мкм, $s_{1}=0.25$ мкм, $s_{2}=0.1$ мкм, $d=25$ нм. В рассматриваемой графеновой структуре возможно возбуждение так называемых „подзатворных“ плазмонов, волновые вектора которых квантуются как $k_{x}=\pi n / w_{l}$ (для $\left.l=1,2\right)$, где $w_{l}$ - ширины различных затворных электродов в элементарной ячейке и $n$ - целое число. В пространственно симметричной структуре только радиационные плазмонные моды $(\mathrm{c} n=1,3,5, \ldots)$ могут возбуждаться падающей волной. В то время как в пространственно асимметричной структуре возможно возбуждение „нерадиационных“ плазмонных мод (с $n=2,4,6, \ldots)$ [15]. В рассматриваемой графеновой структуре было исследовано преобразование падающей электромагнитной ТГц волны в бегущий плазмон при возбуждении „подзатворных“ плазмонных

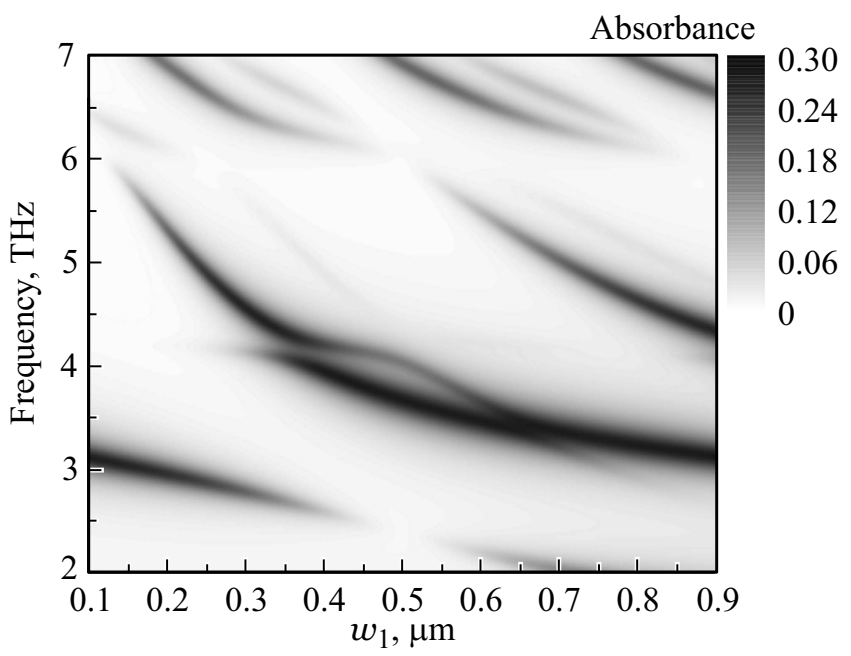

Рис. 2. Спектр коффициента поглощения периодической графеновой структуры $A$ в засимости от ширины затворного электрода $w_{1}$.

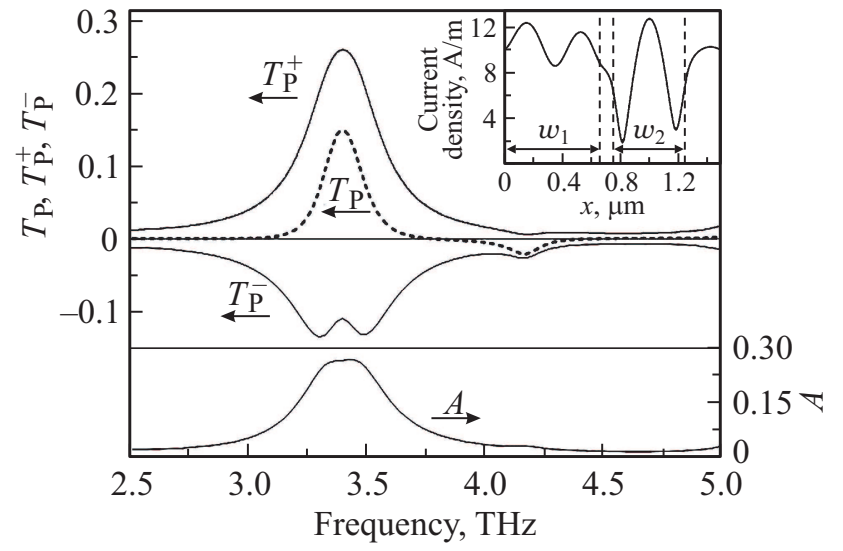

Рис. 3. Спектры коэффициентов преобразования мощности падающей волны в мощность бегущего плазмона $T_{P}, T_{P}^{+}$, и $T_{P}^{-}$при ширине затворного электрода $w_{1}=0.656$ мкм. Кривая коэффициента поглощения $A$ графеновой структуры в нижней части рисунка получена как сечение рис. 2 при $w_{1}=0.656$ мкм. На вставке в верхней части рисунка показано пространственное распределение плотности осциллирующего тока в элементарной ячейке графена в плазмонном резонансе на частоте 3.4 ТГц при $w_{1}=0.656$ мкм.

мод с различными $k_{x}=\pi n / w_{l}$. На рис. 2 показана зависимость спектра коэффициента поглощения в графеновой структуре от ширины затворного электрода $w_{1}$. Плазмонные моды, коэффициент поглощения которых не зависит от $w_{1}$, резонансно возбуждаются под затворным электродом $w_{2}$, и моды, для которых частота обратно пропорциональна ширине затворного электрода $w_{2}$, возбуждаются под затворным электродом $w_{2}$ (рис. 2). Наиболее эффективными с точки зрения увеличения коэффициента преобразования падающей ТГц волны в бегущий плазмон $T_{P}$ являются такие плазмонные резонансы, когда под одним затворным электродом резонансно возбуждается „радиационная“ плазмонная мода с волновым вектором $k_{x}=\pi / w_{2}$, а под другим затворным электродом - „нерадиационная“ плазмонная мода с волновым вектором $k_{x}=\pi 2 / w_{1}$ (на рис. 2 показаны две такие точки): 1) на частоте 3.4 ТГц и $w_{1}=0.656$ мкм и 2) на частоте 4.1 ТГц и $w_{1}=0.36$ мкм). При расчетах произведена оптимизация графеновой структуры для получения наибольшего коэффициента преобразования $T_{P}$ на частоте 3.4 ТГц при $w_{1}=0.656$ мкм по параметрам $w_{2}, d$ и коэффициенту асимметрии $K=1-s_{1} / s_{2}$, при постоянной сумме $s_{1}+s_{2}$.

Максимально достижимый коэффициент преобразования в этом случае составил $15 \%$ для времени релаксации импульса носителей заряда 1 пс (рис. 3) и коэффициента асимметрии $K=0.6$. При возбуждении такого плазмонного резонанса потоки мощности в положительном $T_{P}^{+}$ и отрицательном $T_{P}^{-}$направлении оси $x$ испытывают резонанс и отличаются друг от друга в 2 раза (рис. 3). Пространственное распределение $E_{x}$ компоненты электрического поля в графене для плазмонной моды с 
максимальным коэффициентом преобразования показано на вставке к рис. 3, где показано одновременное возбуждение ,радиационной“ и „нерадиационной“ плазмонных мод.

Максимальное различие между потоками мощности плазмона в положительном $T_{P}^{+}$и отрицательном $T_{P}^{-}$ направлении оси $x$ (рис. 4) можно наблюдать при резонансном возбуждении только „нерадиационной“ подзатворной моды. Графеновая структура была оптимизирована по параметрам $s_{1}, s_{2}, w_{2}, d$ и коэффициенту асимметрии К в целях увеличения коэффициента преобразования мощности в однонаправленно распространяющийся плазмон. На рис. 4 показаны резонансы возбуждения двух „нерадиационных“ мод с волновым вектором

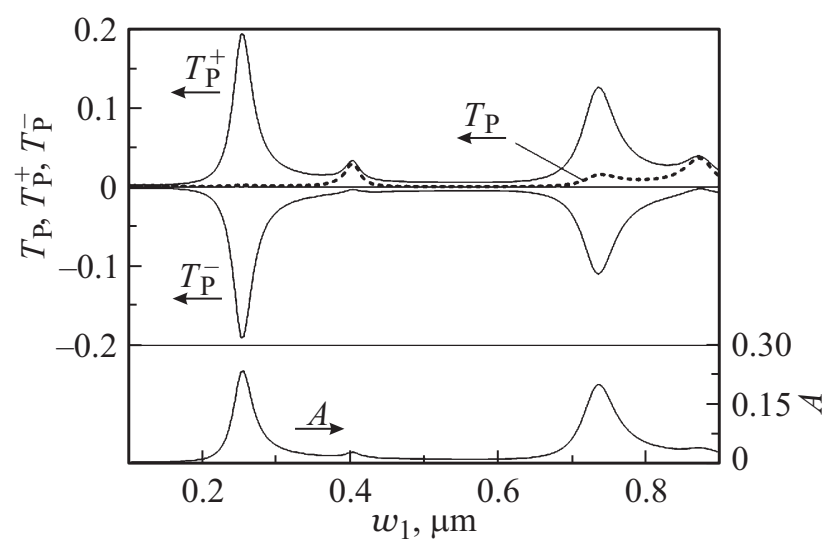

Pис. 4. Зависимость коэффициентов преобразования мощности падающей волны в мощность бегущего плазмона $T_{P}, T_{P}^{+}$и $T_{P}^{-}$от ширины затворного электрода $w_{1}$ на частоте 4.875 ТГц. Кривая коэффициента поглощения $A$ графеновой структуры в нижней части рисунка получена как сечение рис. 2 на частоте 4.875 ТГц.

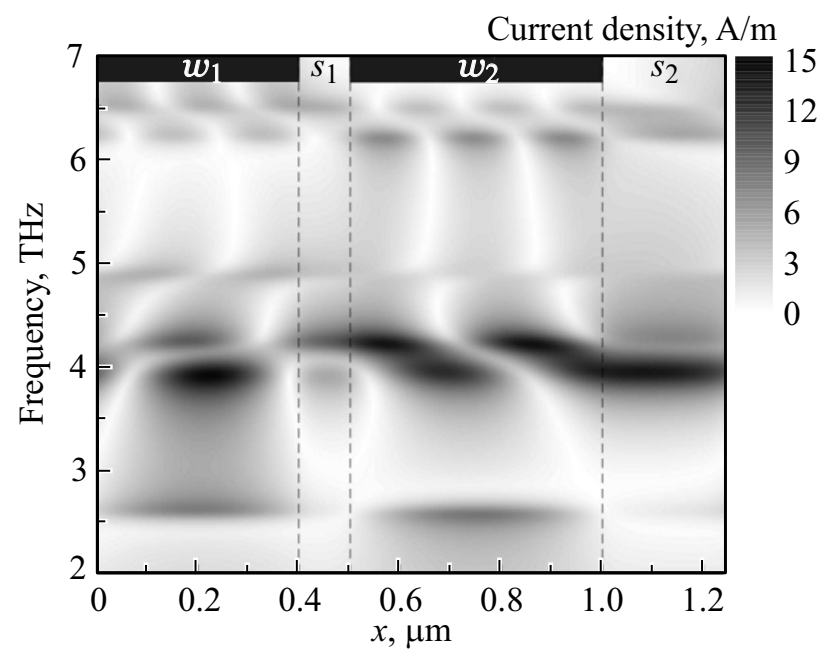

Рис. 5. Пространственное распределение плотности осциллирующего тока в элементарной ячейке графена при резонансном возбуждении „нерадиационной“ плазмонной моды с волновым вектором $k_{x}=2 \pi / w_{1}$ на частоте 4.875 ТГц при $w_{1}=0.404$ мкм.

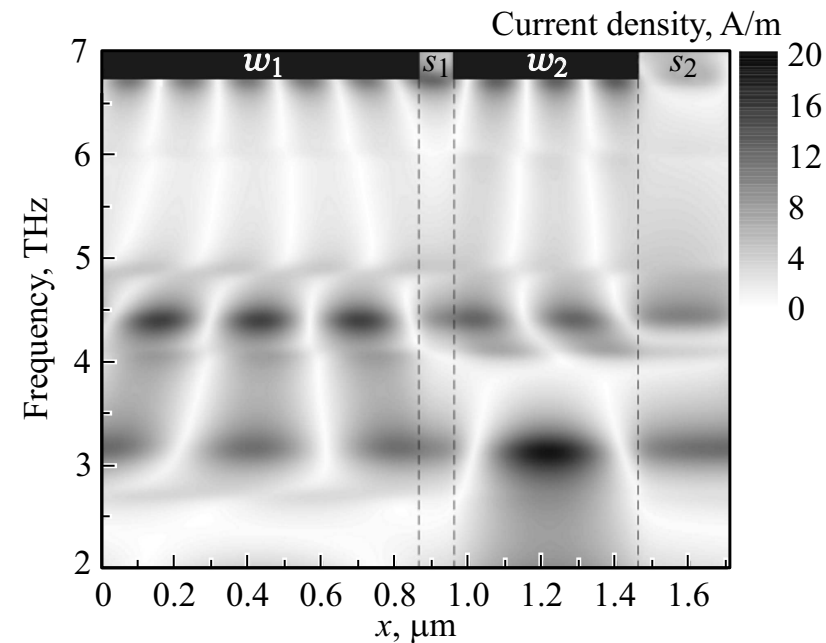

Рис. 6. Пространственное распределение плотности осциллирующего тока в элементарной ячейке графена при резонансном возбуждении „нерадиационной“ плазмонной моды с волновым вектором $k_{x}=4 \pi / w_{1}$ на частоте 4.875 ТГц при $w_{1}=0.872$ мкм.

$k_{x}=2 \pi / w_{1}$ и $w_{1}=0.404$ мкм и волновым вектором $k_{x}=4 \pi / w_{1}$ и $w_{1}=0.872$ мкм на частоте 4.875 ТГц. Максимальное преобразование мощности в однонаправленный плазмон происходит при возбуждении „нерадиационной“ ${ }^{\prime \prime}$ моды с волновым вектором $k_{x}=4 \pi / w_{1}$ (рис. 2 на частоте 4.875 ТГц и $w_{1}=0.872$ мкм) при коэффициенте асимметрии графеновой структуры $K=0.6$ (рис. 4). Выяснено, что в этом случае, несмотря на сравнительно небольшой коэффициент преобразования мощности падающей волны в бегущую мощность плазмона $T_{P}$, разница между потоками мощности плазмона $T_{P}^{+}$и $T_{P}^{-}$может превышать порядок величины (рис. 4 при $w_{1}=0.872$ мкм). Пространственное распределение компоненты электрического поля в графене для „нерадиационной“ плазмонной моды с волновым вектором $k_{x}=2 \pi / w_{1}$ показано на рис. 5 , а для плазмонной моды с волновым вектором $k_{x}=4 \pi / w_{1}$ - на рис. 6 .

\section{4. Заключение}

Таким образом, в данной работе показано, что в периодической графеновой структуре с двойным решеточным затвором и асимметричной элементарной ячейкой возможно возбуждение бегущих плазмонных мод внешней ТГц волной, нормально падающей на плоскость структуры. Наибольший коэффициент преобразования мощности падающей волны в бегущий плазмон достигается при одновременном резонансном возбуждении „радиационной“ и „нерадиационной“ плазмонных мод под разными затворными электродами в элементарной ячейке структуры.

При резонансном возбуждении только „нерадиационной“ плазмонной моды в графеновой структуре мощ- 
ность падающей волны преобразуется в однонаправленно бегущий плазмон. Причем различие между встречными потоками мощности плазмона может составлять больше порядка величины.

\section{Финансирование работы}

Работа поддержана Российским научным фондом, грант № 18-79-10041.

\section{Конфликт интересов}

Авторы заявляют, что у них нет конфликта интересов.

\section{Список литературы}

[1] A.N. Grigorenko, M. Polini, K.S. Novoselov. Nature Photonics, 6, 749 (2012).

[2] F.H.L. Koppens, T. Mueller, P. Avouris, A.C. Ferrari, M.S. Vitiello, M. Polini. Nature Nanotech., 9, 780 (2014).

[3] F.J. García de Abajo. ACS Photonics, 1, 135 (2014).

[4] A.K. Geim, I.V. Grigorieva. Nature, 499, 419 (2013).

[5] D. Svintsov, V. Vyurkov, S. Yurchenko, T. Otsuji, V. Ryzhii. J. Appl. Phys., 111, 083715 (2012).

[6] T. Zhao, S. Gong, M. Hu, R. Zhong, D. Liu, X. Chen, P. Zhang, X. Wang, C. Zhang, P. Wu, S. Liu. Scientific Rep., 5, 16059 (2015).

[7] H. Yan, T. Low, W. Zhu, Y. Wu, M. Freitag, X. Li, F. Guinea, P. Avouris, F. Xia. Nature Photonics, 7, 394 (2013).

[8] B. Wunsch, T. Stauber, F. Sols, F. Guinea. New J. Phys., 8, 318 (2006).

[9] P. Alonso-Gonzalez, A.Y. Nikitin, Y. Gao, A. Woessner, M.B. Lundeberg, A. Principi, N. Forcellini, W. Yan, S. Velez, A.J. Huber, K. Watanabe, T. Taniguchi, F. Casanova, L.E. Hueso, M. Polini, J. Hone, F.H.L. Koppens, R. Hillenbrand. Nature Nanotech., 12, 31 (2017).

[10] N. Kumada, S. Tanabe, H. Hibino, H. Kamata, M. Hashisaka, K. Muraki, T. Fujisawa. Nature Commun., 4, 1363, (2013).

[11] L. Du, D. Tang. J. Optical Soc. America A, 31, 691 (2014).

[12] T. Wenger, G. Viola, J. Kinaret, M. Fogelstrom, P. Tassin. Phys. Rev. B, 97, 085419 (2018).

[13] Y.V. Bludov, M.I. Vasilevskiy, N.M.R. Peres. J. Appl. Phys., 112, 084320 (2012).

[14] A.Y. Nikitin, P. Alonso-Gonzalez, R. Hillenbrand. Nano Lett., 14, 2896 (2014).

[15] V.V. Popov, D.V. Fateev, E.L. Ivchenko, S.D. Ganichev. Phys. Rev. B, 91, 235436 (2015).

[16] D.V. Fateev, K.V. Mashinsky, V.V. Popov. Appl. Phys. Lett., 110, 061106 (2017).

[17] L.A. Falkovsky, A.A. Varlamov. Eur. Phys. J. B, 56, 281 (2007).

[18] M.S. Jang, V.W. Brar, M.C. Sherrott, J.J. Lopez, L. Kim, S. Kim, M. Choi, H.A. Atwater. Phys. Rev. B, 90, 165409 (2014).

Редактор Г.А. Оганесян

\section{Lateral energy transfer by plasmons excited by a terahertz wave in a periodic spatially asymmetric graphene structure}

\author{
D.V. Fateev ${ }^{\mathbf{1}, 2}$, K.V. Mashinsky ${ }^{\mathbf{1}}$, I.M. Moiseenko ${ }^{\mathbf{1}}$, \\ V.V. Popov 1 \\ ${ }^{1}$ Kotelnikov Institute of \\ Radio Engineering and Electronics, \\ Russian Academy of Sciences, \\ 410019 Saratov, Russia \\ 2 Saratov State University, \\ 410012 Saratov, Russia
}

\begin{abstract}
The transformation of the power of terahertz wave normally incident onto a periodic graphene structure into the power of propagating plasmon is studied theoretically. The regime of the maximum transformation of the power of the incident radiation into the power of a traveling plasmon and the regime of the excitation of a unidirectional-traveling plasmon are found. It was shown that up to $15 \%$ of the power of the incident wave can be transformed into a propagating plasmon.
\end{abstract}

